\title{
Parotid duct bleeding, presentation of malignant parotid gland papillary cystadenocarcinoma: rare
}

\section{case report}

\begin{abstract}
Bleeding from the parotid duct as one of the presenting signs and symptoms of the parotid gland carcinoma, is considered rare one in comparison to the other signs and symptoms, like; mass fixation, cervical lymphadenopathy, facial paralysis and skin ulceration. A 47-year-old male patient presented with history of bleeding per mouth associated with right facial swelling, no history of trauma or any surgical intervention. Patient underwent a total parotidectomy and neck dissection after biopsy proven malignancy. Histological examination of the specimen revealed papillary cystadenocarcinoma of the parotid gland with lymph node metastasis. A parotid duct bleeding with parotid mass without history of trauma my considered as clinical sign of parotid gland carcinoma.
\end{abstract}

Volume 10 Issue 4 - 2018

\author{
Omar Al Saraireh, Suhaib Eid \\ Head and Neck Surgery Department, King Hussein Cancer \\ Center, Jordan
}

\begin{abstract}
Correspondence: Omar AI Saraireh, Head and Neck Surgery Department, King Hussein Cancer Center Queen Rania Al Abdullah Street, Jordan, Email ots1976@gmail.com
\end{abstract}

Received: March 07, 2018| Published: August 03, 2018

Keywords: papillary cystadenocarcinoma, parotid gland tumors, signs, parotid duct bleeding, lymph node metastasis

\section{Introduction}

Neck lymphadenopathy, facial nerve palsy, infiltration and ulceration of the overlying skin, pain, rapid tumor growth, tumor fixation and hardness are all considered as an alarming clinical signs and symptoms of parotid gland malignancy, ${ }^{1}$ bleeding from the parotid duct in association with parotid mass is not well known sign of malignancy in the parotid mass. ${ }^{2}$

Papillary cystadenocarcinoma was first described in 1991 by World Health Organization as a separate entity. Papillary cystadenocarcinoma (PCA), is one of the rarest malignancies of the salivary glands, known also as mucin-producing adenopapillary carcinoma, it's a slow growing tumor of the salivary glands. It has a higher incidence in the submandibular gland followed by the parotid gland and minor salivary glands. ${ }^{3}$ It accounts for $2 \%$ of all salivary glands neoplasms.

\section{Case report}

A 46-year-old male patient presented with oral cavity bleeding since three months associated with gradually progressive swelling since four years below and anterior to the right ear (Figure1), the swelling mainly increase in size within one year. No previous history of trauma or radiation, and the family and social history were unremarkable. Physical examination revealed blood draining from the right parotid duct associated with firm, painless, fixed mass, about $10-15 \mathrm{~cm}$ in size, without facial nerve paralysis or other neck masses.

Magnetic resonance imaging (MRI) of the neck revealed a large lobulated mass replacing the superficial lobe of right parotid gland and invades the main parotid duct, without cervical lymph node enlargement Figure2. Features on neck MRI were suggestive of malignant primary salivary gland tumor (likely adenocarcinoma).

Ultrasound-guided soft tissue mass biopsy, 5 passes an $18 \mathrm{G}$ x $20 \mathrm{~cm}$ needle were made into the target mass showed neoplastic cells are positive for Pan-CK, S100, and focally for CK5/6. While they are negative for CD31, P63 and SMA. Mucicarmine highlights intracellular mucin. Perl's stain for iron highlights hemosiderin pigment. Feature of low grade mucoepidermoid carcinoma in right parotid gland. Based on the clinical evaluation and imaging we staged the tumor as T3NOMO

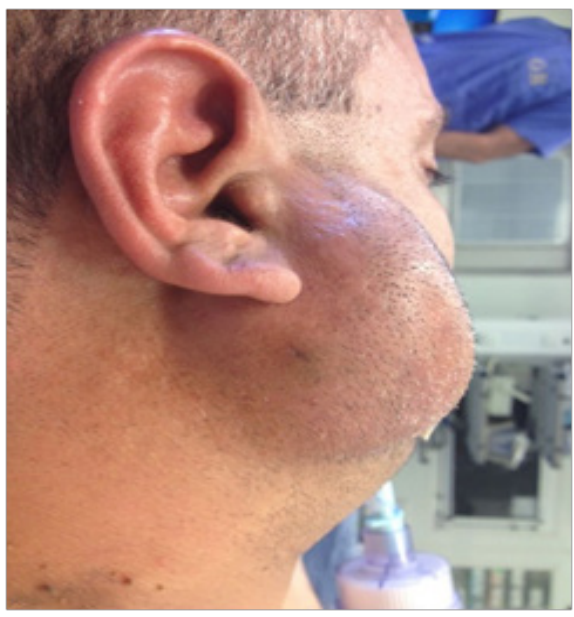

Figure I Facial mass at the right side of the angle of mandible.

Figure 2: Axial Neck MRI Post-contrast fat-saturated T1-weighted images (T1WI) showing a large lobulated mass replacing the superficial lobe of right parotid gland with invasion of the mass into the main parotid duct

Complete resection of the mass with Right total parotidectomy with right supraomohyoid neck dissection were performed. Gross pathological examination revealed the parotid gland tissue measuring $9 \times 6.5 \times 3 \mathrm{~cm}$, with parotid duct measuring $2 \mathrm{~cm}$, sectioning shows encapsulated multilocular cystic and solid lesion measuring $7.5 \times 5$. $5 \times 2.5 \mathrm{~cm}$.

Microscopic examination revealed positive tumor cell for S100 and negative for DOG1, p63, TTF1 and Nose. No extra nodal extension was seen, parotid duct margin was free of tumor, no lymphvascualr invasion, peri-neural invasion was seen (Figure 3). Encapsulated tumor shows cystic changes with area of hemorrhage and extensive papillary formation. Numerous goblet like cells containing mucin were seen, these findings suggested a diagnosis of papillary cystadenocarcinoma of the parotid gland. Without stromal invasion, one lymph nodes showed metastatic tumor. After surgery patient sent for adjuvant radiotherapy. 


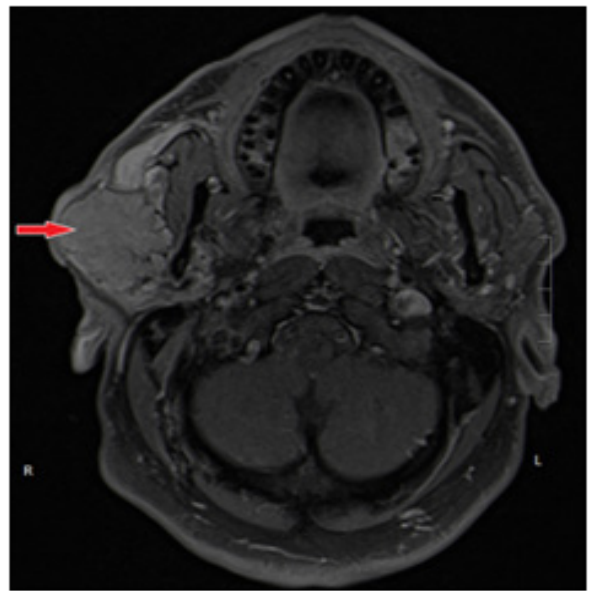

Figure 2 Axial Neck MRI Post-contrast fat-saturated TI-weighted images (TIWI) showing A large lobulated mass replacing the superficial lobe of right parotid gland with invasion of the mass into the main parotid duct.

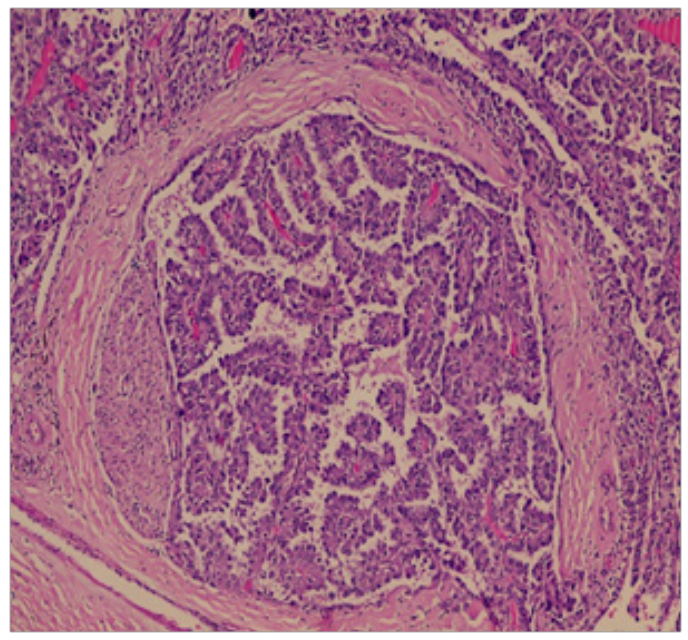

Figure 3 IOX Hematoxylin and Eosin stain shows papillary tumor with perineural invasion and surrounded by fibrous septae.

\section{Discussion}

The data from the literature regarding the Signs and symptoms that alarming the clinician for the possibility of malignancy in the parotid mass are limited, Stodulski et al., ${ }^{2}$ described in details the signs of parotid gland malignancy and there prognostic factors and in his series of 131 patient only tow $(1.5 \%)$ were presented as parotid duct bleeding. ${ }^{4}$ Wong in his paper on symptoms of parotid gland tumors pointed out the following signs, which suggest malignancy: neck lymphadenopathy, facial nerve palsy, infiltration and ulceration of the overlying skin, pain, rapid tumor growth, tumor fixation and hardness. ${ }^{1}$

Salivary gland tumors are very rare diagnoses accounting for 1-6\% of all neoplasms of the head and neck; they have a diverse origin and pathology. ${ }^{5}$ Up to $80 \%$ of the minor salivary glands masses are malignant, where in contrast the vast majority of major salivary gland tumors are benign. ${ }^{6,7}$

Papillary cystadenocarcinoma was first defined in the 1991 by
World health organization, ${ }^{7}$ it is also known as malignant papillary cystadenoma, ${ }^{8}$ low-grade papillary adenocarcinoma, or mucusproducing adenopapillary carcinoma. ${ }^{9}$ It was recognized by WHO as a low-grade malignant tumor with cysts and papillary endophytic projections.

The epithelium is cuboidal or low columnar. Mucus secreting cells can also be present It occupies $2 \%$ of malignant salivary gland masses, with preponderance in the minor salivary glands (about 50\%). It was identified in $45 \%$ of case in the parotid gland and only $5 \%$ in the submandibular gland. ${ }^{10}$ The majority of the patient had a complain of a slow growing ,asymptomatic mass. Despite its character as a low grade tumor, there have been several cases in literature that have presented with an aggressive behavior and higher grade pathological features. ${ }^{11}$ In our case it was associated with lymph node metastasis.

The histological diagnosis of the malignant nature of the tumor is confirmed by nuclear pleomorphism, mitosis and infiltrative growth pattern also The morphological characteristic of the mass is determined by Magnetic resonance imaging which ascertain whether it is solid, necrotic or cystic and its rapports to the other adjacent structures and the degree of infiltration. ${ }^{12}$ Literature review of the management of salivary gland carcinoma is favorable for complete surgical excision which is the main stay of treatment for such cases. ${ }^{13}$

With selective neck dissection being reserved for high stage tumors and/or high grade parotid gland tumor by clinical examination. ${ }^{13,14}$ parotid duct bleeding in association with low grade malignant parotid mass should alarm the clinician toward aggressive behavior of that disease.

Adjuvant radiotherapy is proposed for cases with a large masses $>4 \mathrm{~cm}$, recurrent disease with peri-nueral invasion, nodal disease, incomplete or close margins, and in metastatic disease.

\section{Conclusion}

Bleeding from the parotid duct should alarm the clinician toward more malignant behavior of the parotid mass. Papillary cystadenocarcinoma is a very rare tumor; it's characterized by a low grade, slow growing tumor which is asymptomatic, with the presenting complaint being painless progressive swelling of the affected gland. Clinical signs of advanced TNM stage and parotid duct bleeding as in our case should direct the surgeon toward more aggressive management. ${ }^{15}$

\section{Acknowledgements}

None.

\section{Conflict of interest}

Author declares there is no conflict of interest.

\section{References}

1. Wong DSY. Signs and symptoms of malignant parotid tumours: an objective assessment. J R Coll Surg Edinb. 2001;46:91-95.

2. Stodulski D, Mikaszewski B, Stankiewicz C. Signs and symptoms of parotid gland carcinoma and their prognostic value. Int J Oral Maxillofac Surg. 2012;41(7):801-806.

3. Mardi K, Sharma S, Gupta N. Papillary cystadenocarcinoma of submandibular salivary gland: A rare case report. $J$ Cancer Res Ther. 2010;6(2):330-332. 
4. Kawahara A, Harada H, Mihashi H, et al. Cytological features of cystadenocarcinoma in cyst fluid of the parotid gland: Diagnostic pitfalls and literature review. Diagn Cytopathol. 2010;38(5):377-381.

5. Laurie SA, Licitra L. Systemic therapy in the palliative management of advanced salivary gland cancers. J Clin Oncol. 2006;24(17):2673-2678.

6. Spiro RH. Management of malignant tumors of the salivary glands. Oncology (Williston Park). 1998;12(5):671-680.

7. Iyer NG, Kim L, Nixon IJ, et al. Factors predicting outcome in malignant minor salivary gland tumors of the oropharynx. Arch Otolaryngol Head Neck Surg. 2010;136(12):1240-1247.

8. Seifert G, Sobin LH. Histological classification salivary gland tumors 2nd ed. Berlin: Springer-Verlag; 1991.

9. Nakagawa T, Hattori K, Iwata N, et al. Papillary cystadenocarcinoma arising from minor salivary glands in the anterior portion of the tongue: A case report. Auris Nasus Larynx. 2002;29(1):87-90.
10. Koc M, Yanılmaz M, Yıldırım H, et al. MRI findings of papillary cystadenocarcinoma of the submandibular gland. Diagn Interv Radiol. 2010;16(1):20-23.

11. Pollett A, Perez Ordonez B, Jordan RC, et al. High-grade papillary cystadenocarcinoma of the tongue. Histopathology. 1997;31(2):185-188.

12. Xiaoli Wang, Yijun Luo, Minghuan Li, et al. Management of salivary gland carcinomas-a review. Oncotarget. 2017;8(3):3946-3956.

13. Armstrong JG, Harrison LB, Thaler HT, et al. The indications for elective treatment of the neck in cancer of the major salivary glands. Cancer. 1992;69(3):615-619.

14. Kawata R, Koutetsu L, Yoshimura K, et al. Indication for elective neck dissection for N0 carcinoma of the parotid gland: a single institution's 20-year experience. Acta Otolaryngol. 2010;130(2):286-292.

15. Terhaard $\mathrm{CH}$, Lubsen $\mathrm{H}$, Rasch $\mathrm{CR}$, et al. The role of radiotherapy in the treatment of malignant salivary gland tumors. Int J Radiat Oncol Biol Phys. 2005;61(1):103-111. 